\title{
Is Smoothelin Useful for Detection of Muscularis Propria Invasion in Urinary Bladder Carcinoma Cases?
}

\author{
Nehal Abd El-Ghaffar Heabah", Mohammed Farid Aref, Mohamed Alaa Mokhtar, \\ Mohamed Moustafa Shareef and Ahlam Mohamed Abo El-Enain
}

Pathology Department, Faculty of Medicine, Tanta University, Egypt

*Corresponding author

K e y w o r d s
Urinary bladder
carcinoma,
Smoothelin,
Muscularis propris
invasion

A B S T R A C T

Urinary bladder carcinoma is a common urologic malignancy, particularly in males. We studied the role of smoothelin (a cytoskeleton protein) as a diagnostic marker for muscularis propria invasion, differentiating it from the muscularis mucosae for proper staging in urinary bladder carcinoma patients. Paraffin blocks from 85 cases of bladder carcinoma: 69 urothelial carcinoma cases, 10 squamous cell carcinoma cases, 5 adenocarcinoma cases and one case neuroendocrine small cell carcinoma, were stained by smoothelin immunohistochemical marker. Smoothelin proved an important diagnostic utility, allowing distinction of the muscularis propria MP (positive smoothelin expression) from the muscularis mucosa MM (negative smoothelin expression). The sensitivity and specificity of smoothelin in detecting MP invasion in the current study was $100 \%$. It is important to use smoothelin immunohistochemistry as a routine in all cases of TURBT specimens for accurate staging and subsequent optimal patient management.

\section{Introduction}

Urinary bladder carcinoma (UBC) is an international public health problem (Malats and Real, 2015). It is the $9^{\text {th }}$ frequentlydiagnosed cancer worldwide, the $7^{\text {th }}$ common cancer in men and the $14^{\text {th }}$ leading cause of deaths due to cancer worldwide (Mahdavifar et al., 2016). The highest incidence of bladder cancer is observed in Europe, the United
States and Egypt, respectively, while the lowest level is in Sub- Saharan Africa, Asia and South America, respectively (Antonia et al., 2017). In Egypt, bladder cancer incidence and behavior differ significantly from the developed countries, as bladder cancer has been and remains one of the most prevalent malignancies, accounting for $12.22 \%$ of male cancers and representing the main bulk of the urinary system malignancy (Salem and 
Mahfouz, 2012). Smoothelin is a cytoskeletal protein that is specifically expressed in fully differentiated contractile smooth muscle (Kirna et al., 2015). Smoothelin has been reported to be expressed in the muscularis propria (MP) of normal and overactive bladder (Maake et al., 2006, Paner et al., 2009 and Bovio et al., 2010).

Pathological determination of the depth of invasion is crucial to the accurate staging and subsequent therapy of patients. Namely, tumor invasion limited to the lamina propria (LP) is pathological stage T1 (pT1), whereas tumor extending into the MP is at least pathological stage T2 (pT2) (Vakar-Lopez et al., 2007).

This distinction is critical because invasion of the MP is associated with a poorer prognosis and is one indication for definitive aggressive therapy (e.g. radical cystectomy or chemoradiation) (Humphrey, 2004).

There are several circumstances in which accurate determination of MP invasion may be difficult. The invasion of urothelial carcinoma into the LP often elicits hypertrophy of the muscularis mucosae (MM), changing the appearance from thin wisps of smooth muscle to thickened muscle bundles that can simulate the MP. Also this invasion may induce stromal desmoplastic reaction consisting of myofibroblasts giving false appearance of MP.

This may be particularly problematic in transurethral resection of bladder tumor (TURBT) specimens, in which proper orientation of the material is not possible (Jimenez et al., 2000; Vakar-Lopez et al., 2007).

In addition, carcinomas invading into the MP can often alter the arrangement of the MP muscle by splitting the usually thick muscle bundles, making its recognition difficult (Epstein et al., 2004).

\section{Materials and Methods}

This study was carried out on 85 cases of urinary bladder carcinomas. These cases were selected from the archives of the Pathology department, Faculty of Medicine during the period from January 2017 to December 2018. Approval from the research ethics committee (REC), was taken before conducting the study. The specimens obtained were: 71 specimens of trans-urethral resection of bladder tumors (TURBT) (83.5\%) and 14 radical cystectomy specimens $(16.5 \%)$.

\section{Histopathological study}

Histological sections, 4-mm thick, were stained by hematoxylin and eosin (H\&E) for evaluation of histopathological parameters, including: the histopathological grade, depth of invasion (T), vascular, perineural invasion, associated CIS and whenever possible, lymph node status $(\mathrm{N})$ and prostatic involvement.

The studied urinary bladder carcinoma cases were classified microscopically according to the 2016 World Health Organization (WHO) classification system (Moch et al., 2016).

Pathological Staging of the studied tumors was done according to American Joint Committee on Cancer (AJCC) TNM Pathologic Staging of Urinary Bladder Carcinomas (Edge et al., 2010).

\section{Immunohistochemical staining}

Immunohistochemical staining was performed using the streptavidin-biotin method as described by (Buchwalow and Böcker, 2010), for evaluation of smoothelin expression. From each paraffin block, 4-mm-thick sections mounted onto positively charged slides, deparaffinized and rehydrated. Endogenous peroxidase was blocked by immersion in 3\% hydrogen peroxide. Antigen retrieval using 
microwave oven was performed. The primary antibodies used were:

Smoothelin antibody was a mouse monoclonal antibody raised against cytoskeletal extract from gizzard of chicken origin, (R4A): sc23883 (Santa Cruz Biotechnology, Inc). Two to three drops of smoothelin were placed on each slide at a 1:50 dilution.

\section{Interpretation of smoothelin positivity}

Positive smoothelin staining was indicated by the presence of brownish cytoplasmic staining in the smooth muscle cells of the studied cases

The intensity of smoothelin expression was graded semiquantitatively as follows: Negative (0), Weak (1+), Moderate (2+), or Strong (3+) (Bovio et al., 2010).

\section{Statistical analysis}

The collected data was statistically analyzed using SPSS software statistical computer package version 20. Data were expressed in terms of frequencies (number of cases) and percentages for categorical variables and range, median, mean \pm standard deviation (SD) for continuous variables. For comparing categorical data, Chi-square (X2) test was used as a test of significance. Fisher's exact test or Monte Carlo was used when one or more of cells have an expected frequency of five or less. P values of $<0.05$ were considered statistically significant (Petrie and Sabin, 2005).

\section{Results and Discussion}

\section{Histopathological examination of the studied cases}

The 85 studied cases (78 cases were males, $91.8 \%$ and 7 cases were females, $8.2 \%$ with age ranged between 38 and 86 years, mean age was 61.08), were categorized into 4 groups:

Group I: 69 cases of urothelial carcinoma (UC) $(81.2 \%)$ : including 10 cases of noninvasive low grade papillary UC and 59 cases of infiltrating UC. Infiltrating urothelial carcinomas included: 29 case pure UC, 13 cases UC with squamous differentiation, 4 cases UC with glandular differentiation, 3 cases sarcomatoid UC, 2 cases plasmacytoid UC, 2 cases microcystic UC, 2 cases micropapillary UC, 2 cases clear cell UC, one case UC with both squamous and glandular differentiation, and one case poorly differentiated urothelial carcinoma.

UC cases were graded into: 18 cases low grade urothelial carcinoma (26\%) and 51 cases high grade urothelial carcinoma (74\%). Regarding muscularis propria (MP) invasion in urothelial carcinoma group: 23 cases were NMI "Ta \& T1" (33.3\%) and 46 cases were MI “T2, T3 \& T4" (66.7\%).

Group II: 10 cases of squamous cell carcinoma (SCC) (11.8\%).

Eight cases were moderately differentiated SCC and two cases were poorly differentiated SCC.

Group III: 5 cases of moderately differentiated adenocarcinoma (5.9\%).

Group IV: One case of neuroendocrine small cell carcinoma (SmCC) $(1.1 \%)$, confirmed by strong membranous immunoreactivity to CD56 neuroendocrine marker.

\section{Smoothelin immunohistochemical results}

Out of our 85 cases, muscularis propria (MP) invasion was detected in 62 cases $(72.9 \%)$, while no muscle invasion was found in 23 cases $(27.1 \%)$. 
Table.1 Smoothelin expression in the muscularis propria among the four studied groups

\begin{tabular}{|c|c|c|c|c|c|c|}
\hline \multicolumn{2}{|l|}{ Group } & \multicolumn{3}{|c|}{ Smoothelin Scoring } & \multirow{2}{*}{$\begin{array}{c}\text { Total } \\
\text { positive }\end{array}$} & \multirow[t]{2}{*}{ Total } \\
\hline & & $\begin{array}{c}\text { No } \\
\text { Muscle } \\
\text { Invasion }\end{array}$ & $\begin{array}{l}\text { Moderate } \\
\quad+2\end{array}$ & $\begin{array}{c}\text { Strong } \\
+3\end{array}$ & & \\
\hline \multirow{2}{*}{$\begin{array}{l}\text { Urothelial } \\
\text { carcinoma }\end{array}$} & $\mathbf{N}$ & 23 & 6 & 40 & 46 & 69 \\
\hline & $\%$ & $33.3 \%$ & $8.7 \%$ & $58 \%$ & $66.7 \%$ & $100 \%$ \\
\hline \multirow{2}{*}{$\begin{array}{l}\text { Squamous cell } \\
\text { carcinoma }\end{array}$} & $\mathbf{N}$ & 0 & 0 & 10 & 10 & 10 \\
\hline & $\%$ & $0 \%$ & $0 \%$ & $100 \%$ & $100 \%$ & $100 \%$ \\
\hline \multirow[t]{2}{*}{ Adeno carcinoma } & $\mathbf{N}$ & 0 & 0 & 5 & 5 & 5 \\
\hline & $\%$ & $0 \%$ & $0 \%$ & $100 \%$ & $100 \%$ & $100 \%$ \\
\hline \multirow{2}{*}{$\begin{array}{l}\text { Neuroendocrine } \\
\text { carcinoma }\end{array}$} & $\mathbf{N}$ & 0 & 0 & 1 & 1 & 1 \\
\hline & $\%$ & $0 \%$ & $0 \%$ & $100.0 \%$ & $100 \%$ & $100 \%$ \\
\hline \multirow[t]{2}{*}{ Total } & $\mathbf{N}$ & 23 & 6 & 56 & 62 & 85 \\
\hline & $\%$ & $27.1 \%$ & $7.1 \%$ & $65.9 \%$ & $72.9 \%$ & $100 \%$ \\
\hline
\end{tabular}

Fig.1 Muscularis propria (MP) in case of high grade infiltrating urothelial showing strong cytoplasmic smoothelin expression (+3) (Streptavidin biotin $\mathrm{x} 400)$

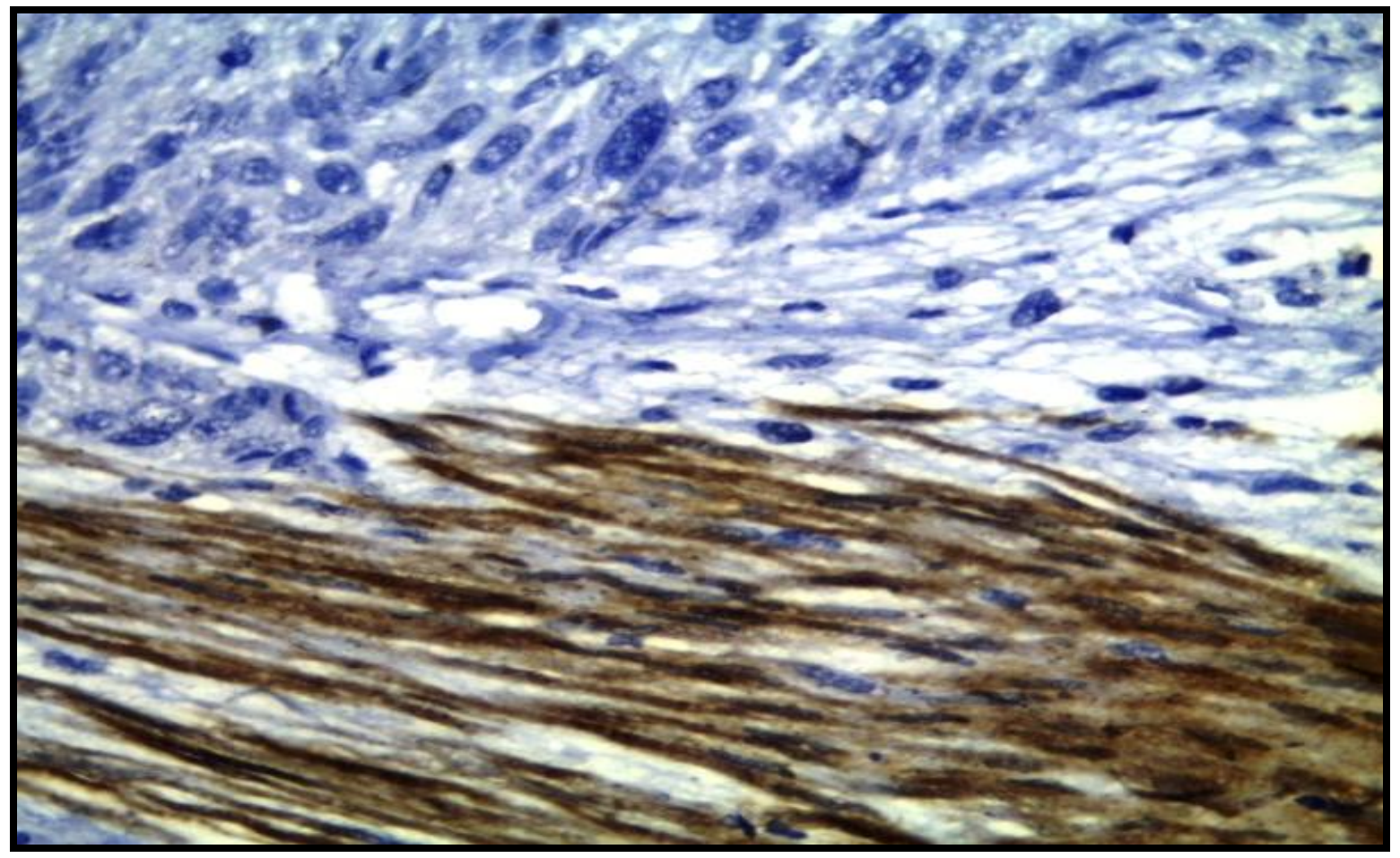


Fig.2 Muscularis propria (MP) in a case of moderately differentiated SCC showing strong cytoplasmic smoothelin expression of the MP (+3) (Streptavidin biotin x400)

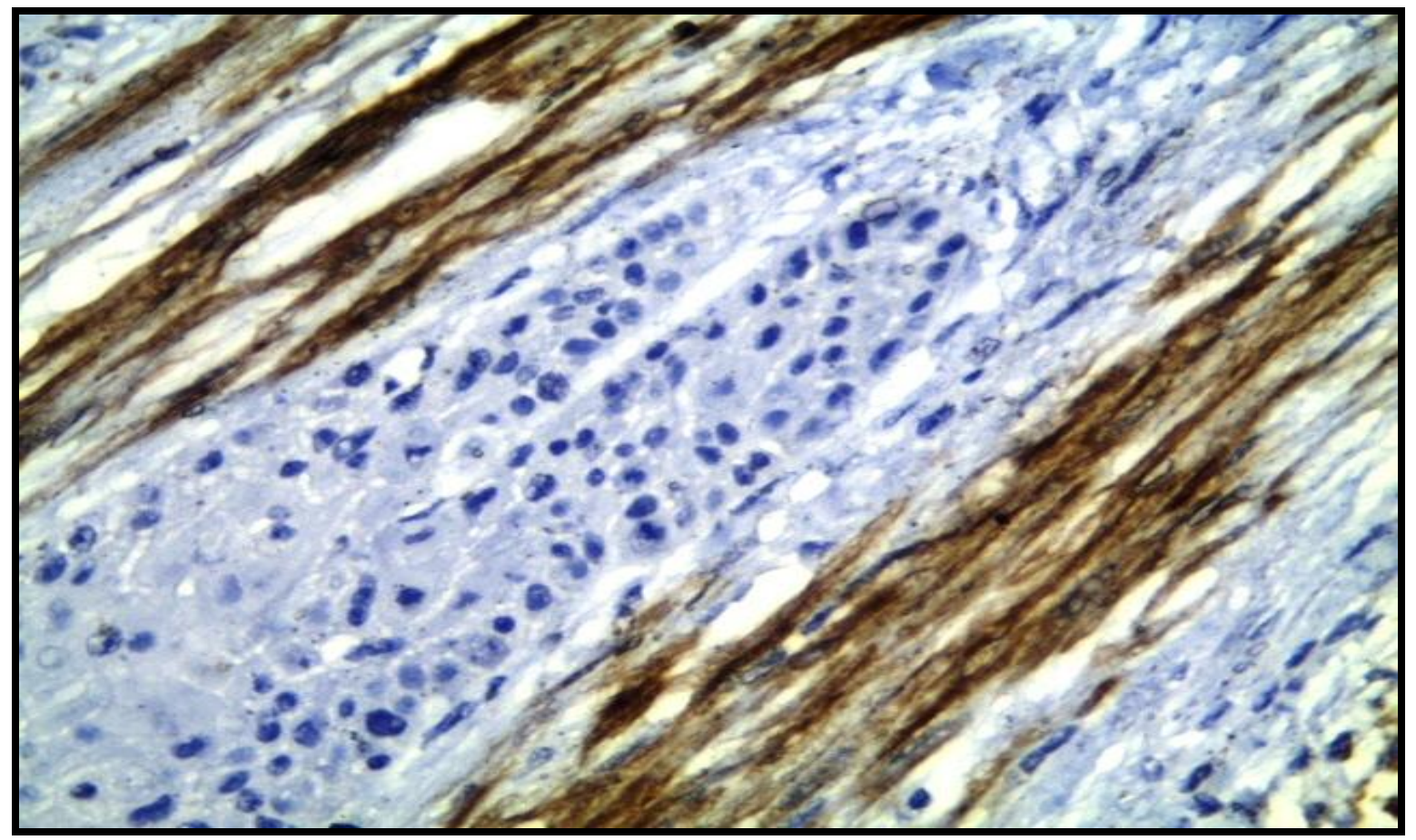

Fig.3 Muscularis mucosa (MM) in a case of high grade infiltrating urothelial carcinoma showing negative smoothelin expression (Streptavidin biotin $\mathrm{x} 400$ )

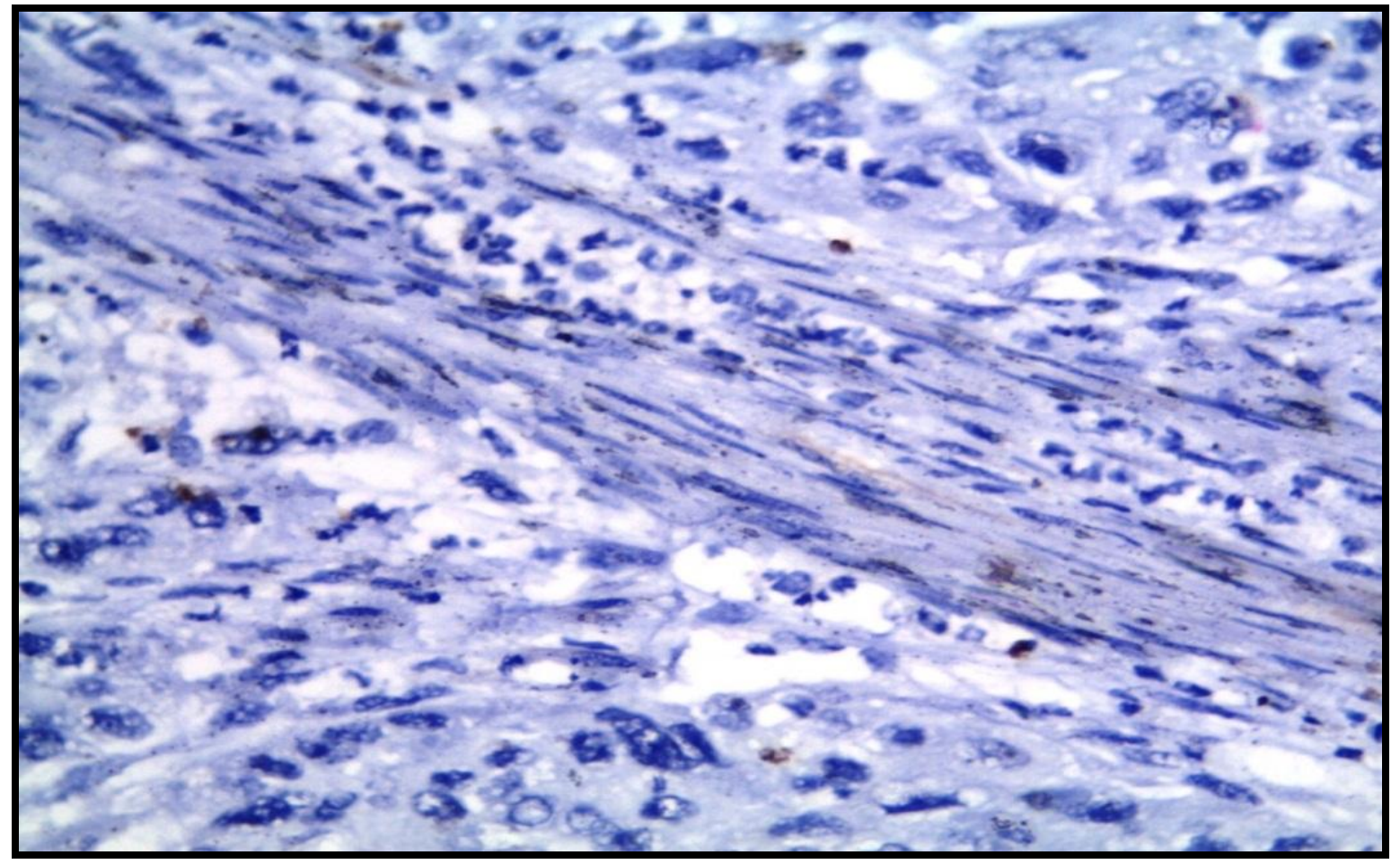


Positive cytoplasmic smoothelin expression was present in the smooth muscle cells of the MP in all of the studied cases. All cases with MP invasion (62 cases) showed positive cytoplasmic smoothelin expression (100\%) with varying intensity, as 56 cases showed strong +3 smoothelin expression, while 6 cases showed moderate +2 smoothelin intensity (Table 1, Figure 1 and 2).

No case of detected MP showed negative or weak staining for smoothelin.

Regarding smoothelin expression in the muscularis mucosa (MM) which was detected as thin wispy muscle bundles, all detected cases showed negative smoothelin expression (Figure 3).

The smooth muscle of the blood vessel walls in the LP showed moderate smoothelin expression (+2) which served as internal control for smoothelin immunohistochemical staining.

\section{Sensitivity and specificity of smoothelin as diagnostic marker for MP invasion}

Considering strong and moderate intensity as positive smoothelin staining, the sensitivity and specificity for smoothelin in MP detection (vs. MM) were $100 \%$. In the current study, all cases with MP invasion showed positive cytoplasmic smoothelin expression (as $90.3 \%$ showed strong +3 smoothelin expression, while $9.7 \%$ showed moderate +2 smoothelin intensity). No case of detected MP showed negative or weak staining for smoothelin.

Regarding smoothelin expression in the muscularis mucosa (MM) which was detected as thin wispy muscle bundles, all detected cases showed negative smoothelin expression. No case of the detected MM showed any reactivity for smoothelin.
The results of this study were in agreement with Kamel et al., (2015) who found that smoothelin showed strikingly different immunoreactivity between MM and MP. In their study, the MM showed absent $(73 \%)$ or weak staining $+1(25 \%)$, only one case showed moderate positive staining +2 (1.6\%), in this case the MP had strong +3 reactivity and none showed strong staining $(0 \%)$. In contrast to the MM, the MP predominantly showed strong staining $(60 \%)$ and moderate staining $(40 \%)$, with none showing weak or negative staining (0\%). Council and Hameed (2009), Paner et al., (2009), Bovio et al., (2010), Gladell et al., (2010) \& El-Osaily et al., (2015) also found that the MP of all evaluated cases showed moderate or strong smoothelin expression. Absent or focal staining with smoothelin was not observed in the MP in any of their studied cases, with all $\mathrm{MM}$ of their cases showing negative or weak expression.

Kirna et al., (2015) also reported that smoothelin Immuno-histochemistry corrected their diagnosis in $3 / 70$ cases that were wrongly diagnosed as invasive (florid desmoplastic response was mistaken for MP). Hence these 3 cases were down-staged after smoothelin IHC.

On the contrary among non-invasive tumors in their study, there were 5 cases which were proven invasive based on smoothelin IHC. These 5 cases were up-staged after IHC. They concluded that it was important to use smoothelin IHC as a routine in all cases of TURBT specimens for proper staging.

The sensitivity and specificity of smoothelin in detecting MP invasion in the current study was $100 \%$. This is in agreement with Council and Hameed, (2009), Kamel et al., (2015) who reported $100 \%$ sensitivity and $100 \%$ specificity for smoothelin in detecting MP invasion. 
Our results were slightly higher than that of Bovio et al., (2010) who stated that smoothelin had a sensitivity of $92 \%$ for detecting MP and a specificity of $97 \%$ for distinguishing between MP and MM.

Elkady et al., (2017) also reported that the intensity of smoothelin expression showed significant difference between MM and MP with $97.5 \%$ sensitivity and $95 \%$ specificity.

They conclude that combined moderate to strong smoothelin and negative vimentin offered $100 \%$ sensitivity and $100 \%$ specificity towards the identification of MP.

Roberts et al., (2014) used smoothelin immunohistochemistry in colorectal carcinoma and reported that all sections of the colonic MP (100\%) had diffuse, strong +3 immunoreactivity for smoothelin while the desmoplastic areas of these tumors, composed of spindled fibroblasts and myofibroblasts, showed negative immune-staining for smoothelin. So smoothelin can help in the accurate staging of cancer and subsequent optimal patient management.

In contrast to the current study and all the previous studies, Poletajew et al., (2017) highlighted a 32\% incidence of moderate smoothelin expression in cases of hyperplastic MM, and as many as $25 \%$ of cases, the reaction intensity within $\mathrm{MM}$ and $\mathrm{MP}$ was similar, concluding that the use of smoothelin immunohistochemistry as a diagnostic tool for MP invasion requires caution and that no single marker is reliable for differentiating between MM and MP, so they suggested to use a combination of anti-smoothelin, and anti-vimentin antibodies for diagnostic purposes.

This discrepancy in the results may be due to different staining methodology and interobserver variability.
The relatively distinct immunohistochemical staining pattern of smoothelin between MP and MM with $100 \%$ sensitivity and specificity, makes it a useful marker to be incorporated in the routine TURBT assessment, could potentially reduce the number of cases that are re-staged, helps in the accurate staging of bladder carcinoma and subsequent optimal patient management.

We haven't submitted this work elsewhere before and the manuscript had been read and approved by all the authors.

\section{Declaration of Conflicting Interests}

The author(s) declared no potential conflicts of interest with respect to the research, authorship, and/or publication of this article.

\section{Funding}

The author(s) received no financial support for the research, authorship, and/or publication of this article.

\section{References}

Antonia S, Ferlaya J, Soerjomatarama I, et al., 2017. Bladder Cancer Incidence and Mortality: A Global Overview and Recent Trends. Eur Urol. 71(1): 96108.

Bovio IM, Al-Quran SZ, Rosser CJ, et al., 2010. Smoothelin immunehistochemistry is a useful adjunct for assessing muscularis propria invasion in bladder carcinoma. Histopathology. 56, 951-956.

Buchwalow IB, Böcker W. 2010. Immunostaining enhancement. In: Immunohistochemistry: Basics and Methods. Ch: 6, pub: Springer-Verlag Berlin Heidelberg. P: 47-60.

Council LN, Hameed O. 2009. Myofibroblasts vs. smooth muscle cells 
and muscularis propria: a potential role for immunohistochemistry in staging bladder carcinoma. Mod Pathol. 22: 639-650.

Edge SB, Byrd DR, Compton CC, et al., 2010. eds.: AJCC Cancer Staging Manual. AJCC: Urinary bladder. $7^{\text {th }}$ edition. New York, NY: Springer, 15:497-505.

Elkady N, Abdou AG, Kandil M, et al., 2017. Diagnostic Value of Smoothelin and Vimentin in Differentiating Muscularis Propria from Muscularis Mucosa of Bladder Carcinoma. Int J Biol Markers. 32(3):e305-e312.

El-Osaily O, Abd El-Aziz G, Bassyoni O. 2015. Prognostic Role of CD44, Smoothelin and MUC1 in Urinary Bladder Carcinoma. Med J Cairo Univ. 83(1):449-458.

Epstein JI, Amin MB, Reuter VE. Invasive urothelial carcinoma. In Epstein JI. Amin MB., Reuter VE. eds. 2004. Biopsy interpretation serie. Bladder biopsy interpretation. Philadelphia, PA: Lippincott, Williams \& Wilkins. 75-99.

Gladell P, Jeffrey B, Shawn L, et al., 2010. Diagnostic use of antibody to smoothelin in the recognition of muscularis propria in transurethral resection of urinary bladder tumor (TURBT) specimens. Am J Surg Patho. 34(6):792-9.

Humphrey PA. 2004. Urinary bladder pathology 2004: an update. Ann Diagn Pathol. 8: 380-9.

Jimenez RE, Keane TE, Hardy HT, et al., 2000. pT1 urothelial carcinoma of the bladder: criteria for diagnosis, pitfalls, and clinical implications. Adv Anat Pathol. 7:13-25.

Kamel N, Helmy N, Hassanein A, et al., 2015. The diagnostic utility of antibodies to smoothelin and desmin in staging of urothelial carcinoma. E J Pathol. 35: 14-23.
Kirna P, Malik A, Sivaramakrishna B, et al., 2015. Role of immune-histochemistry in differentiating muscularis mucosae from muscularis propia as an aid to accurate staging of invasive urothelial carcinoma: a pilot study. Adv Lab Med Int. 5(1): $4-15$.

Maake C, Landman M, Wang X, et al., 2006. Expression of smoothelin in the normal and the overactive human bladder. J Urol. 175(3): 1152-1157.

Mahdavifar N, Ghoncheh M, Pakzad R, et al., 2016. Epidemiology, Incidence and Mortality of Bladder Cancer and their Relationship with the Development Index in the World. Asian Pac J Cancer Prev. 17(1):381-386.

Malats N, Real FX. 2015. Epidemiology of Bladder Cancer. Hematology/ oncology clinics of North America, 29:177-89.

Moch H, Humphrey PA, Ulbright TM, Reuter V. 2016. The 2016 WHO Classification of Tumours of the Urinary System and Male Genital Organs-Part B (84-133): Prostate and Bladder Tumours. (International Agency for Research on Cancer, Lyon, France, Eur Urol, 2016.

Paner GP, Shen SS, Lapetino S, et al., 2009. Diagnostic utility of antibody to smoothelin in the distinction of muscularis propria from muscularis mucosae of the urinary bladder: a potential ancillary tool in the pathologic staging of invasive urothelial carcinoma. Am J Surg Pathol. 33: 9198.

Petrie BM, Sabin GT. 2005. Basis and clinical Biostastics $3^{\text {rd }}$ ed, Lang Medical Book. 43(7): 220.

Poletajew S, Wolińska E, Wasiutyński A, et al., 2017. Immunohistochemical differentiation between muscularis mucosae and muscularis propria for improving the staging of bladder cancer in patients undergoing transurethral 
resection of bladder tumours. Pol J Pathol. 68(3): 218-224.

Roberts JA, Waters L, Ro JY, et al., 2014. Smoothelin and caldesmon are reliable markers for distinguishing muscularis propria from desmoplasia: a critical distinction for accurate staging colorectal adenocarcinoma. Int J Clin Exp Pathol. 7(2): 792-796.

Salem HK, Mahfouz S. 2012. Changing patterns (age, incidence, and pathologic types) of schistosoma-associated bladder cancer in Egypt in the past decade. Urology. 79: 379-83.

Vakar-Lopez F, Shen SS., Zhang S. 2007. Muscularis mucosae of the urinary bladder revisited with emphasis on its hyperplastic patterns: a study of a large series of cystectomy specimens. Ann Diagn Pathol. 11: 395-401.

\section{How to cite this article:}

Nehal Abd El-Ghaffar Heabah, Mohammed Farid Aref, Mohamed Alaa Mokhtar, Mohamed Moustafa Shareef and Ahlam Mohamed Abo El-Enain. 2019. Is Smoothelin Useful for Detection of Muscularis Propria Invasion in Urinary Bladder Carcinoma Cases? Int.J.Curr.Microbiol.App.Sci. 8(07): 350-358. doi: https://doi.org/10.20546/ijcmas.2019.807.043 